\title{
Analysis and Design of Current Regulators Using Complex Vectors
}

Fernando Briz, Member, IEEE, Michael W. Degner, Associate Member, IEEE, and Robert D. Lorenz, Fellow, IEEE

\begin{abstract}
The analysis and design of current regulators for multiphase ac loads is presented using complex vector notation. The use of complex vector notation provides a way of comparing the performance of controller topologies through their complex vector root locus and complex vector frequency-response functions. Limitations in the performance of the synchronous frame proportional plus integral current regulator are outlined and several ways of improving its performance are suggested and investigated.
\end{abstract}

Index Terms-Complex vector controls analysis, complex vector frequency response, complex vector root locus, current regulators, synchronous frame regulators.

\section{INTRODUCTION}

$\mathbf{T}$ HE synchronous frame proportional plus integral (PI) current regulator has become the standard for current regulation of multiphase ac machines due to its capability of regulating ac signals over a wide frequency range [1], [2]. In a reference frame synchronous with the fundamental excitation, the fundamental excitation becomes a dc quantity that is easily regulated to the desired value using a PI controller. Even though the performance characteristics of the synchronous frame PI current regulator may seem intuitive, the multiple-input/multiple-output nature of the system makes performance evaluation difficult. In particular, the standard matrix or scalar notation does not easily lend itself to classical control design techniques such as root locus and frequency-response function (FRF).The synchronous frame PI current regulator can be modeled using both a nonlinear system representation [2] where the synchronous frequency $\omega_{e}$ is considered a system variable or as a linear system (Fig. 1) where $\omega_{e}$ is considered a time-varying system param-

Paper IPCSD 99-106, presented at the 1997 Industry Applications Society Annual Meeting, New Orleans, LA, Ocotober 5-9, and approved for publication in the IEEE TRANSACTIONS ON INDUSTRY APPLICATIONS by the Industrial Power Converter Committee of the IEEE Industry Applications Society. Manuscript submitted for review July 1, 1997 and released for publication January 22, 2000. This work was supported by the University of Oviedo, Ford Motor Company, and Wisconsin Electric Machines and Power Electronics Consortium (WEMPEC), University of Wisconsin, Madison.

F. Briz is with the Department of Electrical, Computer and Systems Engineering, University of Oviedo, E-33208 Gijón, Spain (e-mail: fernando@ hecate.etsiig.uniovi.es).

M. W. Degner is with Ford Research Laboratory, Ford Motor Company, Dearborn, MI 48121-2053 USA (e-mail: mdegner@ ford.com).

R. D. Lorenz is with the Department of Mechanical Engineering and the Department of Electrical and Computer Engineering, University of Wisconsin, Madison, WI 53706 USA (e-mail: lorenz@engr.wisc.edu).

Publisher Item Identifier S 0093-9994(00)04394-2.

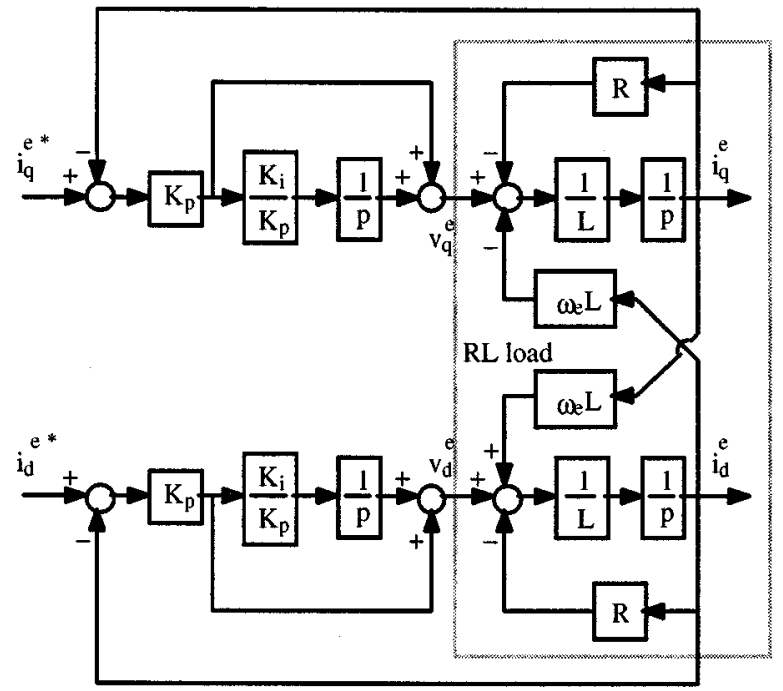

Fig. 1. Scalar block diagram of an $R L$ load with a synchronous frame PI current regulator, shown in the synchronous reference frame.

eter. Modeling $\omega_{e}$ as a parameter allows the use of linear systems tools with the realization that $\omega_{e}$ is assumed to be essentially constant.

The use of complex vector notation simplifies the model of an ac machine from a multiple-input/multiple-output system to an equivalent single-input/single-output complex vector system. The performance of this complex vector model can then be evaluated using generalized forms of the root locus and FRF, the complex vector root locus, and complex vector FRF. The primary goal of this paper is the development of these complex vector design and analysis tools using current regulators as a baseline example.

This paper systematically studies the performance of synchronous reference frame current regulators. First the performance of the synchronous frame PI current regulator is analyzed using scalar notation. An $R L$ load is used initially due to its simple structure and reduced complexity. From this analysis, it will be shown that the performance of this regulator is less than would be intuitively expected. Complex vector modeling will then be introduced as a way of explaining the performance degradation and used to suggest several ways for improvement. Finally, the analysis for the $R L$ load will be extended to the case of ac machine current control. 
TABLE I

$R L$ LOAD PARAMETERS

\begin{tabular}{cc}
\hline Parameter & Value \\
\hline$R$ & $1.1 \Omega$ \\
$L$ & $3.7 \mathrm{mH}$ \\
\hline
\end{tabular}

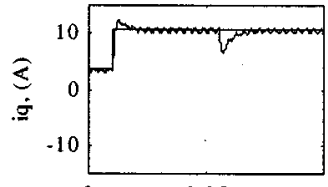

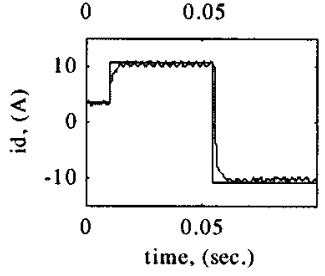

(a)

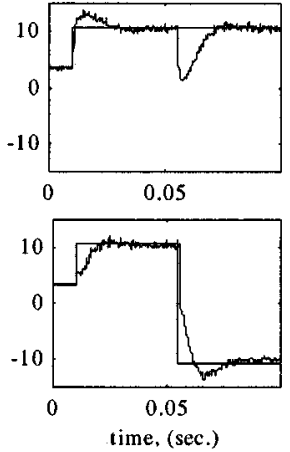

(b)
Fig. 2. Commanded and experimental $q$ - and $d$-axes current step responses for an $R L$ load with a synchronous frame PI current regulator of $200-\mathrm{Hz}$ bandwidth, shown in the synchronous reference frame. (a) $f_{e}=50 \mathrm{~Hz}$. (b) $f_{e}=200 \mathrm{~Hz}$.

\section{SCALAR ANALYSIS OF SYNCHRONOUS FRAME PI CURRENT REGULATORS}

Using scalar notation, the $d q$ model for a symmetric threephase $R L$ load in the stationary reference frame is

$$
\left[\begin{array}{c}
v_{q}^{s} \\
v_{d}^{s}
\end{array}\right]=\left[\begin{array}{cc}
R & 0 \\
0 & R
\end{array}\right]\left[\begin{array}{l}
i_{q}^{s} \\
i_{d}^{s}
\end{array}\right]+\left[\begin{array}{cc}
L & 0 \\
0 & L
\end{array}\right] p\left[\begin{array}{c}
i_{q}^{s} \\
i_{d}^{s}
\end{array}\right]
$$

where $p$ is the derivative operator.

Transforming this model to a reference frame synchronous with the fundamental excitation results in

$$
\left[\begin{array}{c}
v_{q}^{e} \\
v_{d}^{e}
\end{array}\right]=\left[\begin{array}{cc}
R & \omega_{e} L \\
-\omega_{e} L & R
\end{array}\right]\left[\begin{array}{c}
i_{q}^{e} \\
i_{d}^{e}
\end{array}\right]+\left[\begin{array}{cc}
L & 0 \\
0 & L
\end{array}\right] p\left[\begin{array}{c}
i_{q}^{e} \\
i_{d}^{e}
\end{array}\right] .
$$

Adding a synchronous frame PI current regulator to the $R L$ load results in the block diagram shown in Fig. 1.

From (2) and Fig. 1, it can be seen that, in the synchronous reference frame, the $R L$ load has two complex conjugate poles located at $-R / L \pm j \omega_{e}$. The classical synchronous frame PI current regulator adds two open-loop poles to the system located at the origin (the integrators) and two real zeros located at $-K_{i} / K_{p}$.

The performance of the classical synchronous frame PI current regulator was analyzed by applying it to a three-phase $R L$ load with the parameters shown in Table I. The current regulator was tuned by selecting a controller zero approximately equal to the break frequency of the $R L$ load, i.e., $K_{i} / K_{p}=R / L$. The controller gain was selected to achieve a relatively low bandwidth of $200 \mathrm{~Hz}$ that exaggerates the control loop's inherent frequency dependency. Low current regulator bandwidths are used throughout this paper to emphasize the performance characteristics of the various controller topologies studied. Higher bandwidths in almost all cases will reduce these differences.
An overlay of the commanded and experimental system response to step changes in the $q$ and $d$-axis current commands is shown in Fig. 2 for constant input synchronous frequencies of 50 and $200 \mathrm{~Hz}$.

From Fig. 2, it can be seen that, for a low synchronous frequency, $50 \mathrm{~Hz}$, the system behaves nearly as expected for a $200-\mathrm{Hz}$ current regulator bandwidth. For a high synchronous frequency, $200 \mathrm{~Hz}$, serious degradation in the transient performance can be seen. The system responds with characteristics much closer to the time constant of the plant $L / R$ than to the tuned bandwidth of $200 \mathrm{~Hz}$. In addition, significant cross coupling between the responses of the $q$ - and $d$-axes currents exist.

The cause of this performance variation is the frequency dependent degradation in the pole/zero cancellation of the classical synchronous frame PI current regulator. As the synchronous frequency increases, the plant poles and controller zeros migrate further apart, reducing the overall system performance. Complex vector modeling can be used to gain substantial design insight into this frequency-dependent performance degradation.

\section{COMPLEX Vector MOdeling}

The dynamic models for polyphase ac systems can be represented using either complex vector or scalar notation [3]-[5]. The mapping of complex vector to scalar notation is shown in

$$
f_{q d}=f_{q}-j f_{d}
$$

where $f$ represents a generic complex vector quantity.

The complex vector itself can be represented in two forms: 1) Cartesian, as shown in (3), and 2) polar, as shown in

$$
f_{q d}=F e^{j \theta}
$$

where $F=\left|f_{q d}\right|$ and $\theta=a \tan \left(-f_{d} / f_{q}\right)$.

Even though both scalar and complex vector representations give the same results, each has its advantages and disadvantages in analyzing a system. One of the advantages of using complex vectors is that they reduce the order and the number of system inputs and outputs by one-half. For example, the complex vector FRF shows in a single plot the information contained in four FRF's using scalar notation. In general, it is easier to see the system characteristics in one complex vector FRF rather than in four separate scalar FRF's. As a result, the complex vector model is often more suitable for analysis using classical root locus, frequency-response, transfer function, etc., methods.

\section{ANALySIS OF SynCHRONOUS FrAME PI CURRENT REGULATORS USING COMPLEX VECTORS}

Using complex vector notation, the model for an $R L$ load in the stationary reference frame is shown in

$$
v_{q d}^{s}=R i_{q d}^{s}+L p i_{q d}^{s}
$$

and can be transformed to a synchronous reference frame by substituting the " $p$ " operator by " $p+j \omega_{e}$ " as shown in

$$
v_{q d}^{e}=R i_{q d}^{e}+L\left(p+j \omega_{e}\right) i_{q d}^{e} .
$$




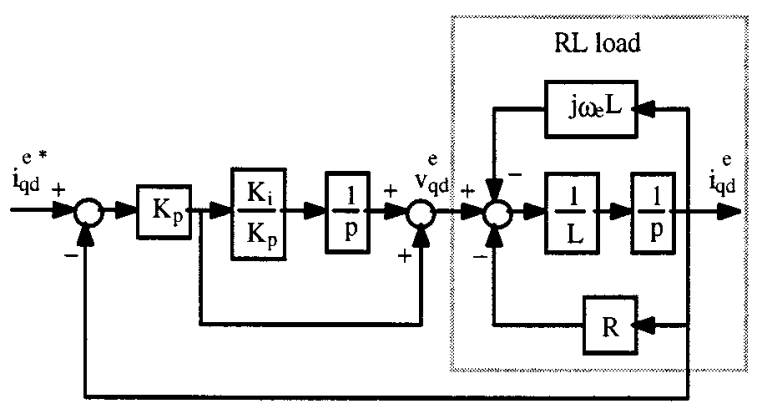

Fig. 3. Complex vector block diagram of an $R L$ load with a synchronous frame PI current regulator, shown in the synchronous reference frame.

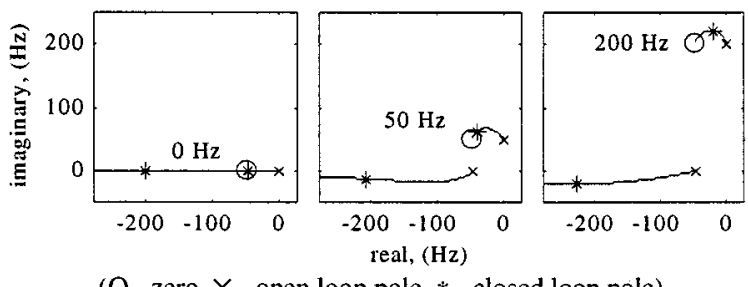

(O - zero, $X$ - open loop pole, $*$ - closed loop pole)

Fig. 4. Complex vector root locus of an $R L$ load with a synchronous frame PI current regulator (200-Hz bandwidth), shown in the stationary reference frame $\left(f_{e}=0,50\right.$, and $\left.200 \mathrm{~Hz}\right)$.

The complex vector representation reduces the order of the system from a second-order to a first-order system. In addition, the system is now a single-input/single-output form, instead of the two-input/two-output form of the scalar model. The complex vector block diagram of the $R L$ load with a synchronous frame PI current regulator is shown in Fig. 3 in a synchronous reference frame.

From (6), it can be seen that, in the synchronous reference frame, the plant has a single asymmetric complex pole located at $-R / L-j \omega_{e}$. Fig. 4 shows the complex vector root locus for three different synchronous frequencies with the current regulator tuned identically to the tuning in the previous section ( $K_{i} / K_{p}=R / L, 200-\mathrm{Hz}$ nominal bandwidth). The root locus was obtained using standard root locus functions in the Matlab controls systems toolbox. The stationary reference frame was considered the best reference frame to show the complex vector root locus and will be used for it throughout this paper.

There are several key observations that can be made from the three root loci plots. First, the complex vector root locus, as scalar root locus, follows the magnitude and angle conditions. However, it does not have to be symmetric with respect to the real axis. This is due to the fact that the inputs and outputs are no longer real numbers but complex vectors and it is possible to get complex asymmetric poles and zeros. Second, the performance degradation of the synchronous frame PI current regulator as the synchronous frequency increases can be deduced as follows.

At low frequencies, the controller zero (a real number zero) approximately cancels the plant pole (when the physical pole is mostly real). This allows the response of the system to be dominated by the faster closed-loop pole, placed at the desired 200-Hz bandwidth.

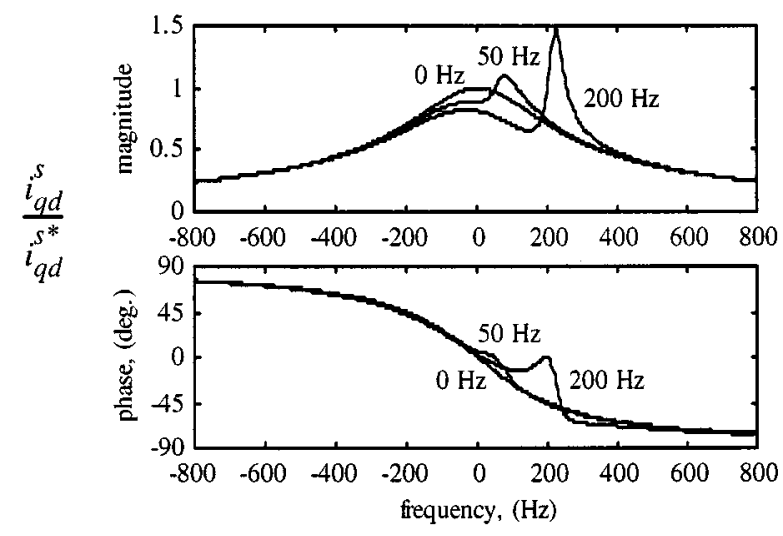

Fig. 5. Complex vector FRF of an $R L$ load with a synchronous frame PI current regulator (200-Hz bandwidth), shown in the stationary reference frame $\left(f_{e}=\right.$ 0,50 , and $200 \mathrm{~Hz}$ ).

At higher synchronous frequencies, the controller zero interacts more with the integration pole added by the controller. The resulting slower root moves progressively closer to the imaginary axis, away from the zero, and increasing overshoot should be expected.

Because the system has a single-input/single-output form when modeled using complex vector notation, a single transfer function completely describes its behavior

$$
\frac{i_{q d}^{s}}{i_{q d}^{s^{*}}}=\frac{K_{p} \cdot s+K_{i}-j K_{p} \omega_{e}}{L s^{2}+\left(K_{p}+R-j \omega_{e} L\right) s+K_{i}-j \omega_{e}\left(K_{p}+R\right)} .
$$

The complex vector FRF, shown in Fig. 5, is calculated from this transfer function. The complex vector FRF is plotted with both positive and negative frequencies since it is possible for complex vectors to rotate both forward (positive frequencies) and backward (negative frequencies). It is important to note that this also requires complex vector FRF's to be plotted using a linear (rather than logarithmic) frequency scale. If the root locus were symmetric about the real axis the FRF would be identical for positive and negative frequencies, but because of the asymmetry shown in the root locus the FRF is asymmetric. The stationary reference frame will be used throughout this paper for complex vector FRF plots.

From Fig. 5, it can be seen that the change in the complex vector FRF as the synchronous frequency increases is rather dramatic. The overshoot and undershoot as the synchronous frequency increases agrees with the behavior shown in the root locus plots. It is important to note that all of the FRF's shown in Fig. 5 have a unity gain and zero phase shift at their synchronous frequencies. However, at frequencies away from the synchronous frequency there is significant distortion in the FRF's.

It is important to understand the meaning of the FRF at frequencies different than the synchronous frequency. The synchronous frequency is the steady-state fundamental component. Both disturbances and changes in the command trajectory will excite the system with a wide range of frequency content. The FRF shows how the system will respond to the frequency content that is not at the steady-state synchronous frequency. 

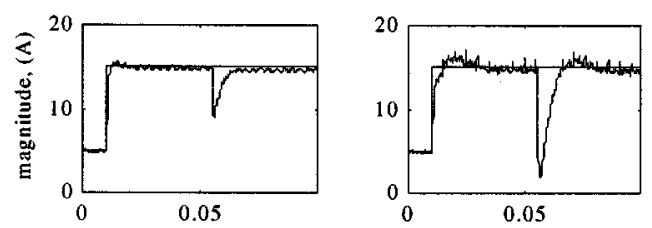

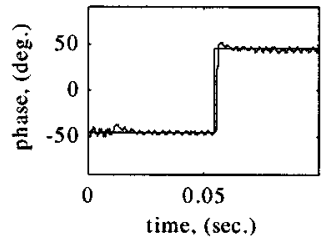

(a)

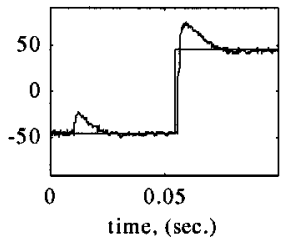

(b)
Fig. 6. Commanded and experimental current magnitude and phase step responses for an $R L$ load with a synchronous frame PI current regulator (200-Hz bandwidth), shown in the synchronous reference frame. (a) $f_{e}=50$ Hz. (b) $f_{e}=200 \mathrm{~Hz}$.

The power of the complex FRF can be seen in Fig. 5, where each individual curve completely describes the system behavior for a single synchronous frequency, while the set of curves clearly shows the dependency of the synchronous PI current regulator on the synchronous frequency.

Complex vector notation can also be used to plot the time response of the system as the polar "magnitude" and polar vector angle ("phase") of the complex vector instead of as the magnitude of the $q$ - and $d$-axes quantities individually. The time response shown in Fig. 2 is replotted in Fig. 6 using the complex vector magnitude and phase. The command trajectory for the time response can be seen to consist of a step change in the magnitude command followed by a step change in the phase command. The synchronous reference frame will be used throughout the remainder of this paper for the time response plots.

Even though the commanded synchronous frequency remained constant for the time responses shown in Fig. 6, both the steps in the magnitude and phase of the commanded current introduce a large set of transient harmonics at frequencies centered on the synchronous frequency. Because of this, the transient response of the current regulator depends on its capability to regulate frequencies other than the synchronous frequency. The FRF's shown in Fig. 5 are consistent with the behavior shown in Fig. 6.

\section{Alternative Design of CURREnt Regulators FOR A THREE-PHASE SYMMETRIC RL LOAD}

The deteriorated time response for the classical synchronous frame PI current regulator has been explained using complex vector FRF and root locus methods. An ideal synchronous reference frame current regulator would have a time response independent of the synchronous frequency when viewed in the synchronous reference frame. Such a regulator would have a complex vector FRF with a shape that does not vary with the synchronous frequency. Instead, the center of the FRF shape would just shift so that it is always symmetric about the synchronous frequency.

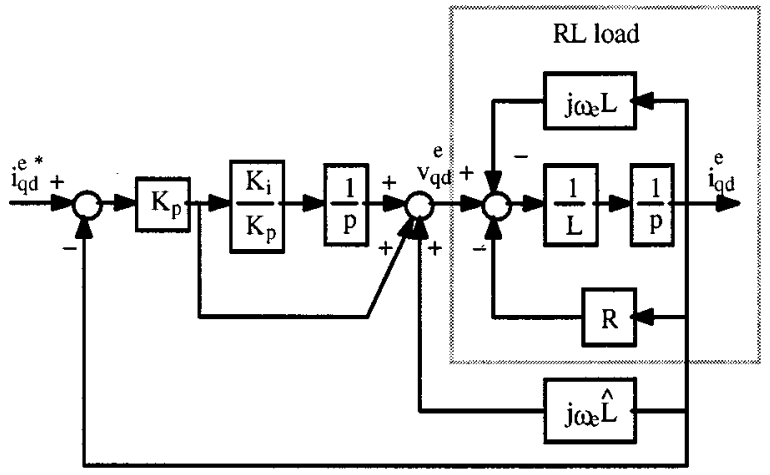

Fig. 7. Complex vector block diagram of an $R L$ load with a cross-coupling decoupling synchronous frame pi current regulator, shown in the synchronous reference frame.

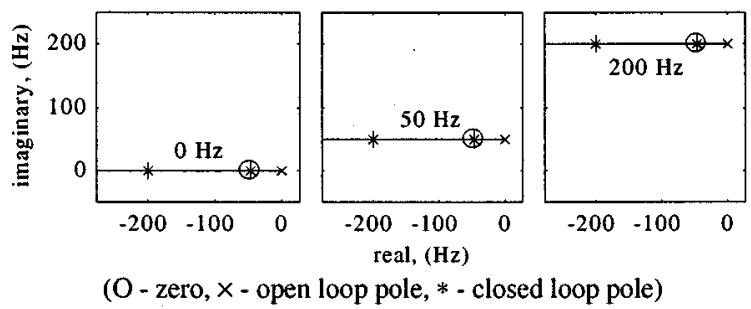

Fig. 8. Complex vector root locus of an $R L$ load with a cross-coupling decoupling synchronous frame PI current regulator $(200-\mathrm{Hz}$ bandwidth), shown in the stationary reference frame $\left(f_{e}=0,50\right.$, and $\left.200 \mathrm{~Hz}\right)$.

\section{A. Synchronous Frame PI Current Regulator With Cross-Coupling Decoupling}

One way of modifying the synchronous frame PI current regulator is to decouple the cross coupling between the $q$ and $d$ axes caused by the $\omega_{e} L$ term in (2), which corresponds to decoupling $j \omega_{e} L$ in (6). Decoupling this term will make the performance of the current regulator independent of the synchronous frequency. The block diagram of the cross-coupling decoupling form of the synchronous frame PI current regulator is shown in Fig. 7 in a synchronous reference frame.

The effect of the cross-coupling decoupling is to move the pole of the plant from $-R / L-j \omega_{e}$ to $-R / L$ in the synchronous reference frame $\left(-R / L\right.$ to $-R / L+j \omega_{e}$ in the stationary reference frame). By moving the pole to this location it is now possible to directly cancel it using the real zero added by the controller. In fact, the PI controller gains necessary to achieve this pole/zero cancellation and the desired bandwidth are identical to those used for the synchronous frame PI current regulator in the previous sections $\left(K_{i} / K_{p}=R / L\right.$, with $K_{p}$ tuned for the desired bandwidth). The resulting complex vector root locus for the cross-coupling decoupling synchronous frame PI current regulator is shown in Fig. 8 for three different synchronous frequencies.

\section{B. Complex Vector Synchronous Frame PI Current Regulator}

The methodology used for the design of the cross-coupling decoupling synchronous frame PI current regulator suggests an alternative way of achieving similar results. The cross-coupling 


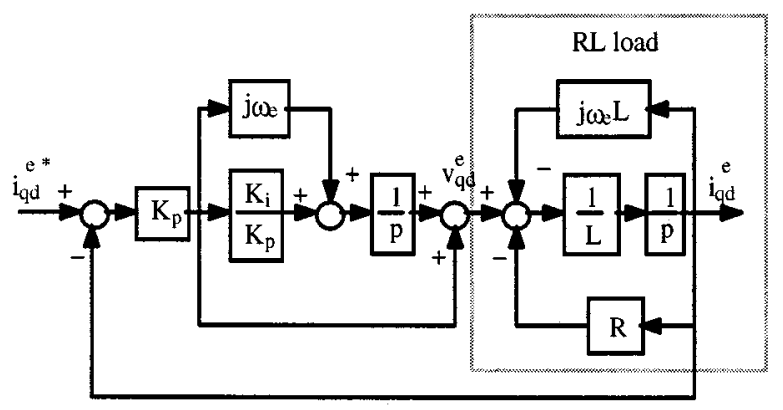

Fig. 9. Complex vector block diagram of an $R L$ load with a complex vector synchronous frame PI current regulator, shown in the synchronous reference frame.

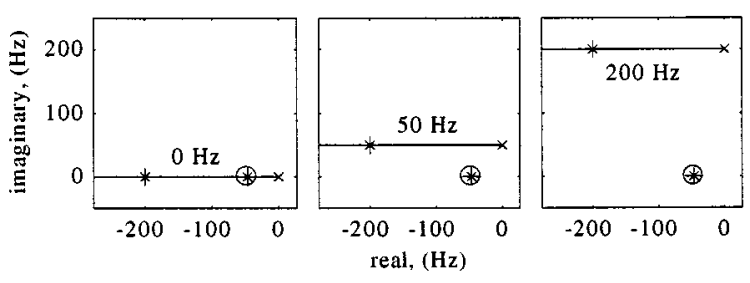

(O - zero, $\times$ - open loop pole, $*$ - closed loop pole)

Fig. 10. Complex vector root locus for an $R L$ load with a complex vector synchronous frame PI current regulator (200-Hz bandwidth), shown in the stationary reference frame $\left(f_{e}=0,50\right.$, and $\left.200 \mathrm{~Hz}\right)$.

decoupling synchronous frame PI current regulator attempted to move the plant pole to the location of the controller zero through the use of decoupling. The dual to this methodology is to move the controller zero to the location of the plant pole by modifying the controller structure. The modified form of the synchronous frame PI current regulator that achieves the desired pole/zero cancellation is shown in Fig. 9. This form of the synchronous frame PI current regulator will be called the complex vector synchronous frame PI current regulator throughout the rest of this paper.

The basic design theory behind the complex vector synchronous frame PI current regulator is to place the zero added by the controller approximately on top of the plant pole, even if the plant pole is complex. This is directly analogous to the classical control pole/zero cancellation methodology, with the only difference being that the use of complex vectors allows the placement of the controller zero off of the real axis. For the case of the RL load this results in the plant pole remaining at $-R / L-j \omega_{e}$ in the synchronous reference frame, or $-R / L$ in the stationary reference frame, with the controller zero placed on top of it. The actual PI controller gains necessary to achieve this pole/zero cancellation and desired bandwidth are identical to the gains used for the two forms of synchronous frame PI current regulators shown in the previous sections ( $K_{i} / K_{p}=R / L$, with $K_{p}$ tuned for the desired bandwidth). The resulting complex vector root locus is shown in Fig. 10 for three different synchronous frequencies.

The current regulators shown in Figs. 7 and 9 are implemented in the synchronous reference frame. Both have an equivalent stationary reference frame implementation that

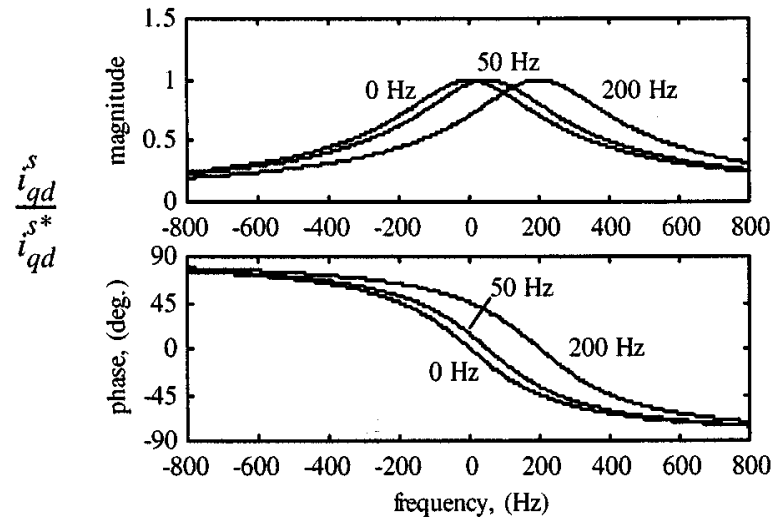

Fig. 11. Complex vector FRF of an $R L$ load for either a cross-coupling decoupling or complex vector synchronous frame PI current regulator $(200-\mathrm{Hz}$ bandwidth), shown in the stationary reference frame $\left(f_{e}=0,50\right.$, and $\left.200 \mathrm{~Hz}\right)$.

is not shown due to space constraints. The two forms of modified synchronous PI current regulators have previously been presented and analyzed using scalar notation [6], [7]. Complex-vector-based analysis will be shown to provide increased insight into their performance characteristics beyond that demonstrated with scalar analysis.

\section{ANALYSis OF CURRENT Regulators FOR AN $R L$ LOAD}

As was done for the conventional synchronous frame PI current regulator, it is possible to calculate the complex vector FRF's for the two modified forms of the synchronous frame PI current regulator. The transfer functions for the cross-coupling decoupling and complex vector forms are shown in (8) and (9), respectively,

$$
\begin{aligned}
\frac{i_{q d}^{s}}{i_{q d}^{s^{*}}} & =\frac{K_{p} s+K_{i}-j K_{p} \omega_{e}}{\left(\begin{array}{r}
L s^{2}+\left(K_{p}+R-j \omega_{e}(L+L)\right) s \\
+K_{i}-\omega_{e}^{2} \hat{L}-j \omega_{e}\left(K_{p}+R\right)
\end{array}\right)} \\
\frac{i_{q d}^{s}}{i_{q d}^{s^{*}}} & =\frac{K_{p} s+K_{i}}{L s^{2}+\left(K_{p}+R-j \omega_{e} L\right) s+K_{i}-j \omega_{e} R} .
\end{aligned}
$$

Although it is not obvious from their transfer functions, if the parameter estimates are correct, and both current regulators have the same PI gains, the complex vector FRF's for the two current regulators are identical and shown in Fig. 11. This agrees with the pole/zero distribution of the two regulators seen in their corresponding root loci plots.

The shape of the complex vector FRF shown in Fig. 11 is independent of the synchronous frequency and symmetric with respect to it. This is identical to the desired FRF.

Fig. 12 shows the step response for the complex vector synchronous frame PI current regulator. (The cross-coupling decoupling controller has nearly identical characteristics and, thus, is not shown.)

The command trajectory goes through the same magnitude and phase variations used to test the conventional synchronous frame PI current regulator in Figs. 2 and 6. The improved performance exhibited by both of the modified synchronous frame 


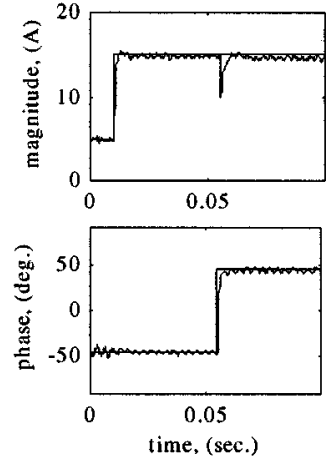

(a)
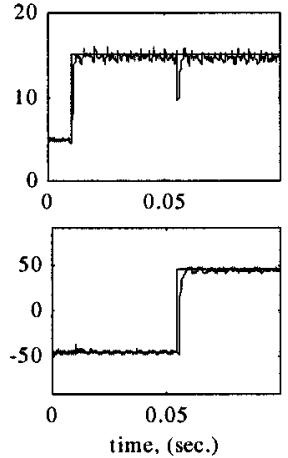

(b)
Fig. 12. Commanded and experimental current magnitude and phase step response for an $R L$ load with a complex vector synchronous frame PI current regulator (200-Hz bandwidth), shown in the synchronous reference frame. (a) $f_{e}=50 \mathrm{~Hz}$. (b) $f_{e}=200 \mathrm{~Hz}$.
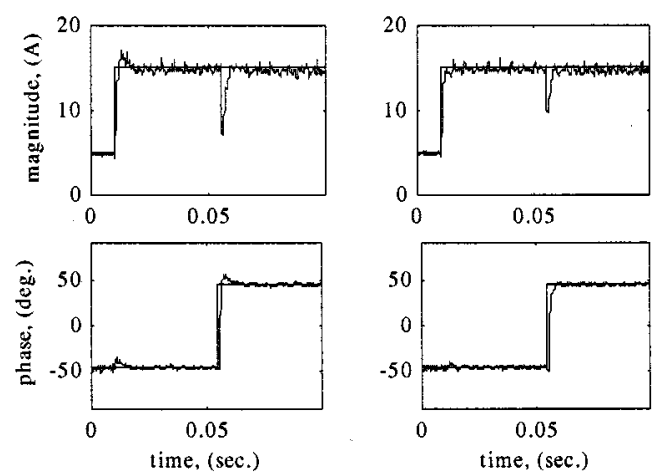

(a)

(b)

Fig. 13. Commanded and experimental current magnitude and phase step responses for $20 \%$ underestimation of $L$ for an $R L$ load with two forms of synchronous frame PI current regulators (200-Hz bandwidth), shown in the synchronous reference frame $\left(f_{e}=200 \mathrm{~Hz}\right)$. (a) Cross-coupling decoupling. (b) Complex vector.

PI current regulators is obvious. Their time responses correspond to the tuned bandwidth independent of the synchronous frequency.

The analysis of the synchronous frame PI current regulators up to this point has assumed that all of the parameters estimates used for tuning are identical to the actual system parameters. A suitable parameter sensitivity analysis will now be presented. It will be limited to the two modified forms of the synchronous frame PI current regulator with the classical synchronous frame PI as benchmark.

Fig. 13 shows the time response for the cross-coupling decoupling and complex vector forms of the synchronous frame PI current regulator, respectively, for $20 \%$ underestimation of $L$ at a synchronous frequency of $200 \mathrm{~Hz}$. Although both current regulators still achieve performance significantly better than the conventional synchronous frame PI current regulator with correct parameter estimates, Fig. 13 shows that the complex vector form of the synchronous frame PI current regulator is less sensitive to errors in $L$.

The reasons for the decreased parameter sensitivity of the complex vector form of the synchronous frame PI current regu-

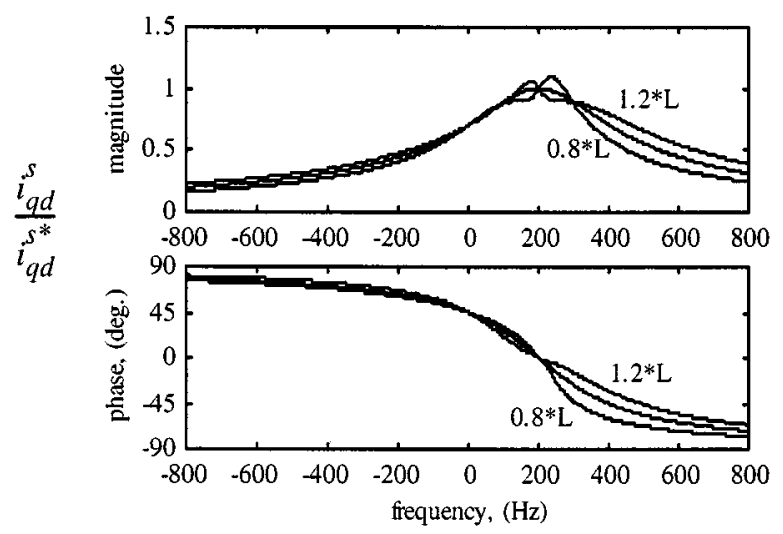

Fig. 14. Complex vector FRF for variations in $L$ of an $R L$ load with a cross-coupling decoupling synchronous frame PI current regulator $(200-\mathrm{Hz}$ bandwidth), shown in the stationary reference frame $\left(f_{e}=200 \mathrm{~Hz}\right)$.

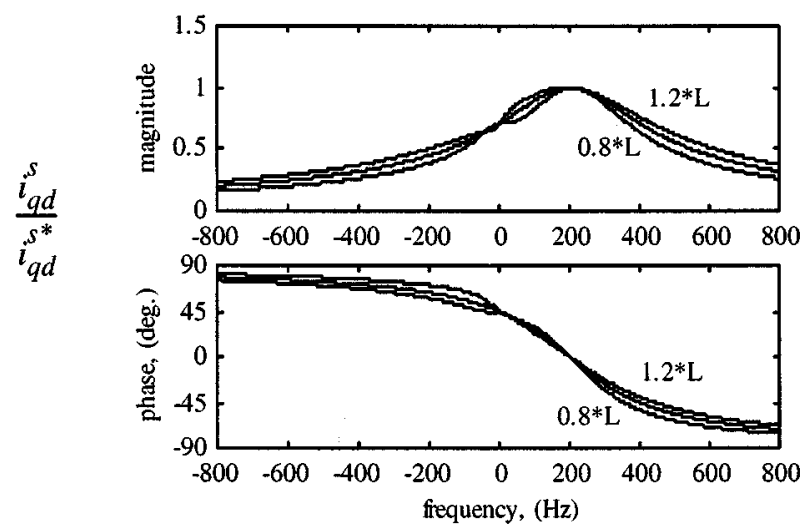

Fig. 15. Complex vector FRF for variations in $L$ of an $R L$ load with a complex vector synchronous frame PI current regulator (200-Hz bandwidth), shown in the stationary reference frame $\left(f_{e}=200 \mathrm{~Hz}\right)$.

lator can be explained using the complex vector root locus. The complex vector synchronous frame PI current regulator achieves the desired pole/zero cancellation by moving the controller zero to the location of the plant pole. Because of this, the approximate pole/zero cancellation always occurs along the real axis of the root locus in the stationary reference frame. When the parameter estimates are incorrect the dynamics of any inexact pole/zero cancellation will be shifted away from the synchronous frequency for any synchronous frequency except dc. The crosscoupling decoupling synchronous frame PI current regulator, on the other hand, achieves the desired pole/zero cancellation by moving the plant pole to the controller zero. This forces the dynamics of any inexact pole/zero cancellation to move with the synchronous frequency.

The complex vector FRF can also be used to analyze the parameter sensitivity of the current regulators. Figs. 14 and 15 show the complex vector FRF's at a synchronous frequency of $200 \mathrm{~Hz}$ for errors in the inductance estimate of the crosscoupling decoupling and complex vector synchronous frame PI current regulators, respectively. The cross-coupling decoupling synchronous frame PI current regulator has the most significant deviations due to parameter errors centered right at the synchronous frequency, while the complex vector synchronous fra- 


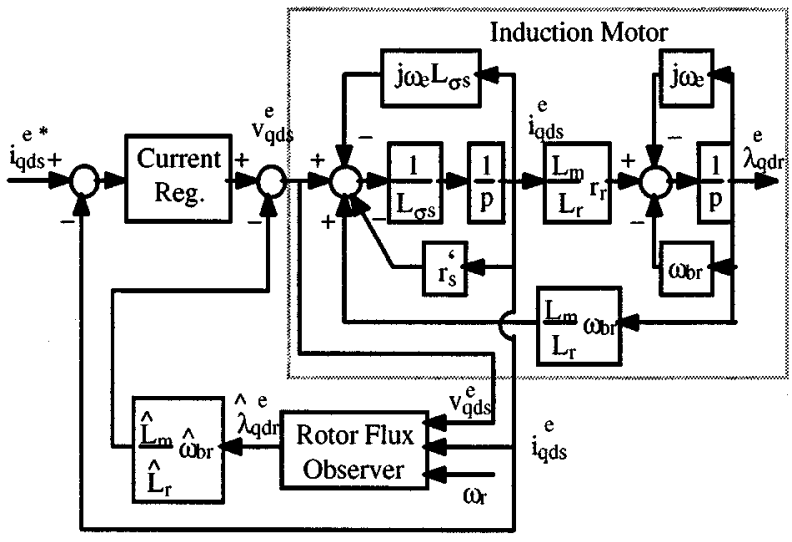

Fig. 16. Complex vector block diagram showing the implementation of a current regulator for a three-phase induction motor using back-EMF decoupling, shown in the synchronous reference frame.

me PI current regulator shifts these deviations away from the synchronous frequency.

\section{DESIGN OF CURRENT REgUlators FOR AC MACHINES}

An $R L$ load was used for the initial discussion of complex vector design methods for synchronous frame current regulators. While this model and method may be appropriate for active filters and uniterruptible power supply (UPS) systems, the analysis will now be extended to the control of current in ac machine drives.

The equations governing the dynamic behavior of an induction motor with the stator current and rotor flux as the state variables are given in (10) and (11) in a synchronous reference frame [5]

$$
\begin{aligned}
& p i_{q d s}^{e}=\frac{1}{L_{\sigma s}}\left(v_{q d s}^{e}-\left(r_{s}^{\prime}+j \omega_{e}\right) i_{q d s}^{e}+\frac{L_{m}}{L_{r}} \omega_{b r} \lambda_{q d r}^{e}\right) \\
& p \lambda_{q d r}^{e}=\frac{L_{m}}{L_{r}} r_{r} i_{q d s}^{e}-\left(\omega_{b r}+j \omega_{e}\right) \lambda_{q d r}^{e}
\end{aligned}
$$

where

$$
L_{\sigma s}=L_{s}-\frac{L_{m}^{2}}{L_{r}} r_{s}^{\prime}=r_{s}+\left(\frac{L_{m}}{L_{r}}\right)^{2} r_{r} \omega_{b r}=\frac{r_{r}}{L_{r}}-j \omega_{r} .
$$

Using these equations, it is possible to transform the induction motor into an equivalent $R L$ load by approximately decoupling the back EMF caused by the rotor flux from the stator equation (10), as shown in Fig. 16.

The decoupling of the back EMF requires an estimate of the rotor flux such as in [4]. Because the cross-coupling decoupling and complex vector synchronous frame PI current regulators basically have the same performance if the parameter estimates are correct, only the classical synchronous frame PI and the complex vector synchronous frame PI current regulators will be shown here.

All of the ac machine current regulator topologies in this paper were tuned for a $200-\mathrm{Hz}$ bandwidth using an induction motor whose parameters are shown in Table II.
TABLE II

INDUCTION MOTOR PARAMETERS

\begin{tabular}{cc}
\hline Parameter & Value \\
\hline$r_{s}^{\prime}$ & $0.82 \Omega$ \\
$L_{\sigma s}$ & $5.5 \mathrm{mH}$ \\
$L_{m}$ & $148 \mathrm{mH}$ \\
$L_{r}$ & $150 \mathrm{mH}$ \\
$R_{r}$ & $0.36 \Omega$ \\
Power Rating & $6 \mathrm{KW}$ \\
Poles & 4 \\
\hline
\end{tabular}
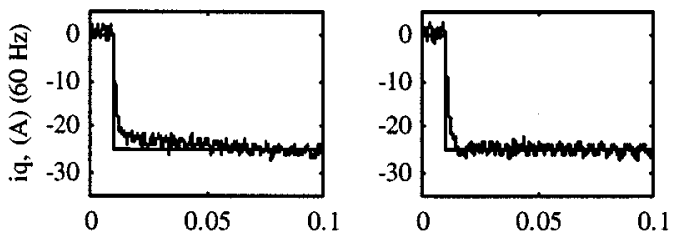

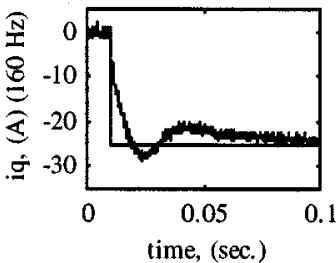

(a)

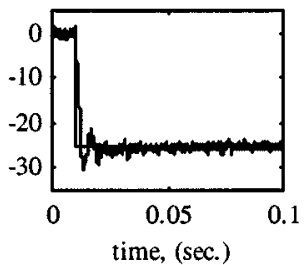

(b)
Fig. 17. Experimental $q$-axis current step response on a rotor-field-oriented induction motor for classical and complex vector synchronous frame PI current regulators with back-EMF decoupling (200-Hz bandwidth) for $f_{e}=60$ and $160 \mathrm{~Hz}$, shown in the synchronous reference frame. (a) Classical synchronous reference frame PI with decoupling. (b) Complex vector synchronous reference frame PI with decoupling.

Fig. 17 shows the experimental step response of the $q$-axis current for two different initial synchronous frequencies, 60 and $160 \mathrm{~Hz}$. The better performance of the complex vector current regulator at both low and high synchronous frequencies can be seen. The results are very similar to those for the $R L$ load with the corresponding controllers.

It is also possible to regulate the current in an ac machine without decoupling the back EMF. The block diagram for this topology is shown in Fig. 18.

The complex vector root locus and FRF for both classical and complex vector synchronous frame current regulators without back-EMF decoupling are shown in Figs. 19 and 20, respectively. Since back-EMF decoupling was not used, a fixed slip must be used in order to relate the rotor speed $\omega_{r}$ in (10) and (11) to the synchronous frequency $\omega_{e}$.

Comparing Fig. 20(a) and (b) with the ideal FRF shown in Fig. 11, it can be seen that the classical synchronous frame PI controller has inferior dynamics at high synchronous frequencies when compared to the complex vector PI.

The comparative experimental step responses for the classical synchronous frame PI and the complex vector PI without back-EMF decoupling are shown in Fig. 21 for a $160-\mathrm{Hz}$ operating frequency. The results are shown for three different control zeros. The experimental step responses for all of the tuning variants show the potential improvement that can be achieved 


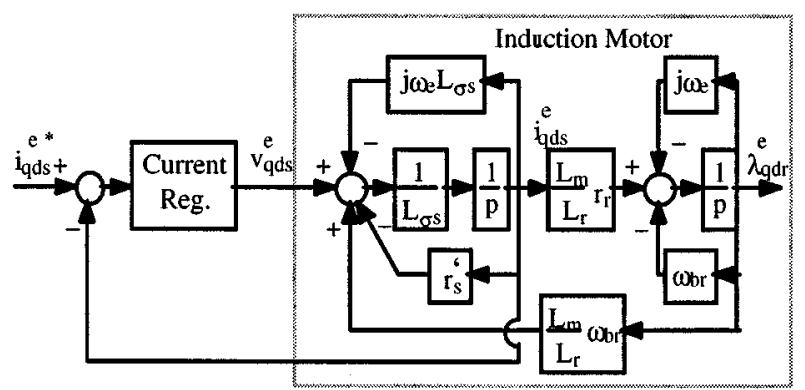

Fig. 18. Complex vector block diagram showing the implementation of a current regulator for a three-phase Induction motor without explicit back-EMF decoupling, shown in the synchronous reference frame.
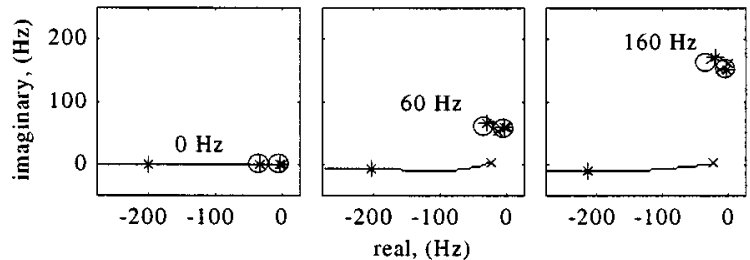

(a)
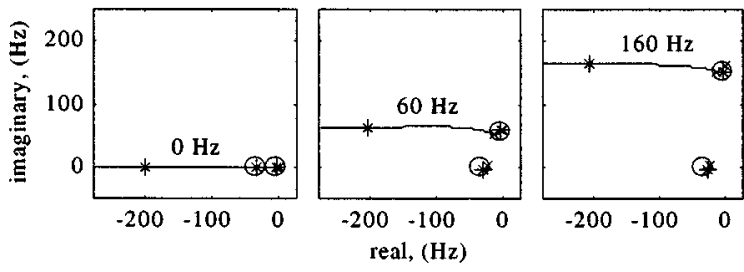

(O - zero, $X$ - open loop pole, $*$ - closed loop pole)

(b)

Fig. 19. Complex vector root locus of a current regulated induction motor at rated slip (200-Hz bandwidth), shown in the stationary reference frame $\left(f_{e}=\right.$ 0,60 , and $160 \mathrm{~Hz}$ ). (a) Classical synchronous frame PI current regulator without decoupling. (b) Complex vector synchronous frame PI current regulator without decoupling.

by use of the complex vector design as compared to the classical design. The low dependency of the complex vector current regulator with respect to the placement of the regulator zero can be seen as an evidence of its low parameter sensitivity.

\section{CONCLUSION}

This paper has attempted to make two contributions: 1) development of complex vector methods for current regulator design and analysis and 2) a comparison of current regulator performance using the developed analysis tools.

It was shown that the used complex vector root locus and FRF's greatly enable controls design.

From the paper's theoretical and experimental results several important conclusions can be reached.

- The performance of the classical synchronous frame PI current regulator degrades as the synchronous frequency approaches the current regulator bandwidth.
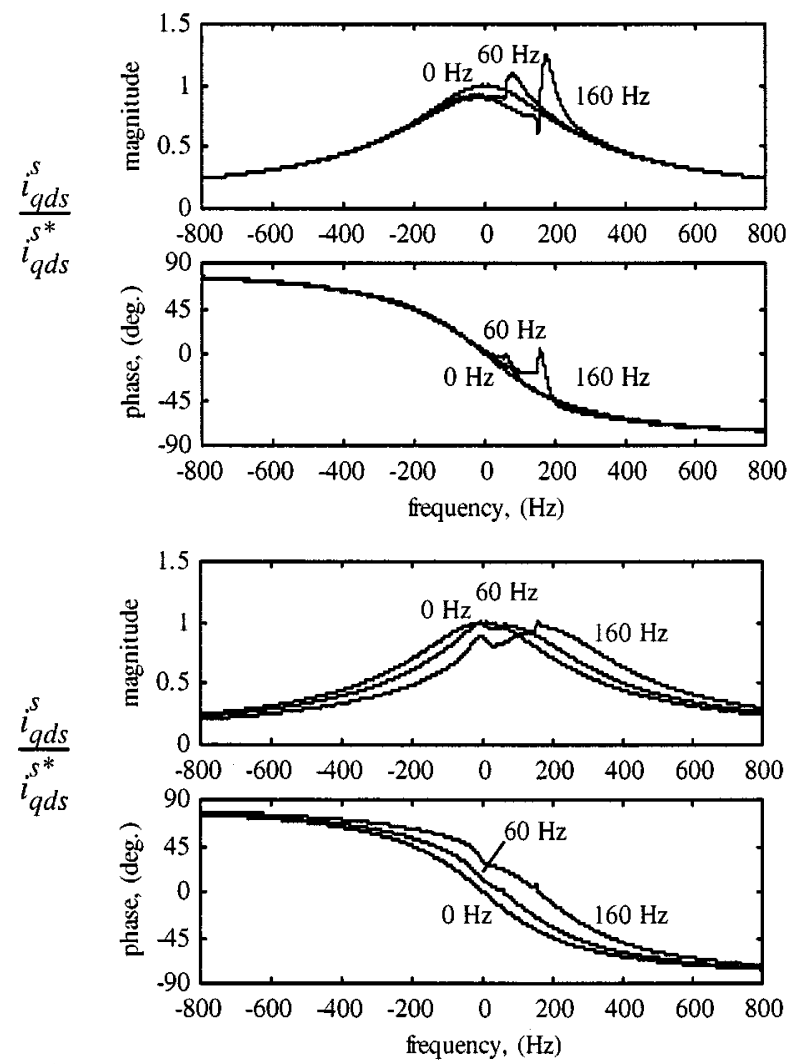

Fig. 20. Complex vector FRF at rated slip on an induction motor with classical and complex vector synchronous frame PI current regulator without back-EMF decoupling (200-Hz bandwidth) for $f_{e}=0,60$, and $160 \mathrm{~Hz}$, shown in the stationary reference frame.
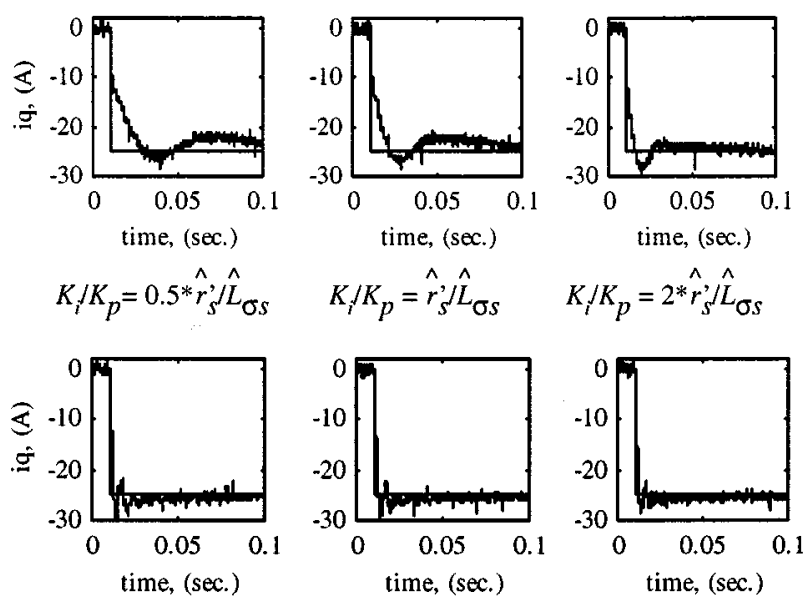

$K_{i} / K_{p}=0.5^{*} \hat{r}_{s}^{\prime} \hat{L}_{\sigma s}$

$K_{i} / K_{p}=\hat{r}_{s}^{s} / \hat{L}_{\sigma s}$

$K_{i} / K_{p}=2 * \hat{r}_{s}^{\prime} \hat{L}_{\sigma s}$

Fig. 21. Experimental $q$-axis current step response for a rotor-field-oriented induction motor with classical and complex vector synchronous frame PI current regulator (without decoupling), 200-Hz bandwidth, for $f_{e}=160 \mathrm{~Hz}$, shown in the synchronous reference frame.

- The performance degradation occurs even if the controller zero is exactly equal to the physical (electrical) time constant. Synchronous frame PI tuning methods other than pole/zero cancellation exhibit similar degradation as the synchronous frequency increases since the asymmetric 
complex vector root locus and FRF are intrinsic to its structure.

- The complex vector synchronous frame PI design implements a more desirable pole/zero cancellation. Even with incorrect parameter estimates, it exhibits little dependency on the synchronous frequency.

- The additional computational requirements of the improved synchronous reference frame current regulators are minimal.

\section{REFERENCES}

[1] C. D. Schauder and R. Caddy, "Current control of voltage-source inverters for fast four-quadrant drive performance," IEEE Trans. Ind. Applicat., vol. IA-18, pp. 163-171, Mar./Apr. 1982.

[2] T. R. Rowan and R. L. Kerman, "A new synchronous current regulator and an analysis of current-regulated PWM inverters," IEEE Trans. Ind. Applicat., vol. IA-22, pp. 678-690, July/Aug. 1986.

[3] J. Holtz, "On the spatial propagation of transient magnetic fields in AC machines," IEEE Trans. Ind. Applicat., vol. 32, pp. 927-937, July/Aug. 1996.

[4] P. J. Jansen and R. D. Lorenz, "A physically insightful approach to the design and accuracy assessment of flux observers for field oriented IM drives," IEEE Trans. Ind. Applicat., vol. 30, pp. 101-110, Jan./Feb. 1994.

[5] D. W. Navotny and T. A. Lipo, Vector Control and Dynamics of AC Drives. New York: Oxford Univ. Press, 1996.

[6] L. J. Garces, "Current control of field oriented induction motor drives," in An IEEE Tutorial: Microprocessor Control of Motor Drives and Power Converters. New York: IEEE Press, 1995, pp. 5.1-5.46.

[7] L. Harnefors and H. P. Nee, "Model based current control of AC machines using the internal model control method," IEEE Trans. Ind. Applicat., vol. 34, pp. 133-141, Jan./Feb. 1998.

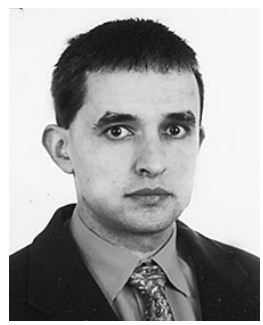

Fernando Briz (A'96-M'99) received the M.S. and $\mathrm{Ph} . D$. degrees from the University of Oviedo, Gijón, Spain, in 1990 and 1996, respectively.

From June 1996 to March 1997, he was a Visiting Researcher at the University of Wisconsin, Madison. He is currently an Assistant Professor in the Electrical Engineering Department, University of Oviedo. His topics of interest include control systems, high-performance ac drives control, sensorless control, and digital signal processing.

Prof. Briz was the recipient of a Prize Paper Award from the Industrial Power Converter Committee of the IEEE Industry Applications Society.

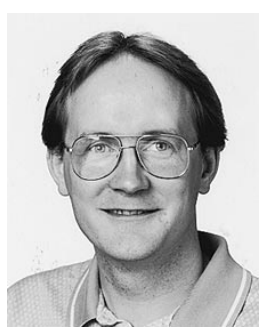

Michael W. Degner (S'95-A'98) received the B.S., M.S., and Ph.D. degrees in mechanical engineering with a focus on electromechanics and control from the University of Wisconsin, Madison, in 1991, 1993 and 1998, respectively.

$\mathrm{He}$ is currently with Ford Research Laboratory, Ford Motor Company, Dearborn, MI, where his research is focused on the use of power electronics in automobile applications. His interests include control systems, machine drives, electric machines, power electronics, and electromechanics.

Dr. Degner received the Second Prize Paper Award from the Industrial Drives Committee and the Third Prize Paper Award from the Industrial Power Converter Committee at the 1997 IEEE Industry Applications Society Annual Meeting.

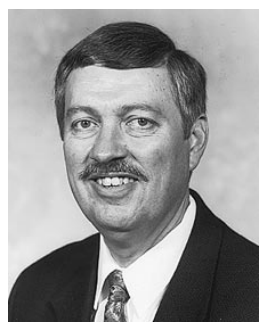

Robert D. Lorenz (S'83-M'84-SM'91-F'98) received the B.S., M.S., and Ph.D. degrees from the University of Wisconsin, Madison, in 1969, 1970, and 1984, respectively.

Since 1984, he has been a Member of the Faculty of the University of Wisconsin, Madison, where he is the Consolidated Papers Foundation Professor of Controls Engineering in both the Mechanical Engineering and Electrical and Computer Engineering Departments. In this position, he acts as Co-Director of the Wisconsin Electric Machines and Power Electronics Consortium. He is also an active consultant to many organizations. He was a Visiting Research Professor in the Electrical Drives Group, Catholic University of Leuven, Leuven, Belgium, in the summer of 1989 and in the Power Electronics and Electrical Drives Institute, Technical University of Aachen, Aachen, Germany, in the summers of 1987, 1991, 1995, 1997, and 1999. From 1969 to 1970, he did his thesis research for the M.S. degree at the Technical University of Aachen. From 1972 to 1982, he was a Member of the Research Staff at the Gleason Works, Rochester, NY. His current research interests include sensorless electromagnetic motor/actuator technologies, real-time signal processing and estimation techniques, precision multiaxis motion control, and ac drive and high-precision machine control technologies.

Dr. Lorenz is currently the IEEE Industry Applications Society (IAS) Vice President/President Elect, a Distinguished Lecturer of the IAS for 2000/2001, the immediate past Chair of the IAS Awards Department, past Chairman of the IAS Industrial Drives Committee, and a member of the IAS Industrial Drives, Electric Machines, Industrial Power Converter, and Industrial Automation and Control Committees. He is a member of the IEEE Sensor Council AdCom and the IEEE Neural Network AdCom. He is a Registered Professional Engineer in the States of New York and Wisconsin. He is also a Member of the American Society of Mechanical Engineers, Instrument Society of America, and Society of Photo-Optical Instrumentation Engineers. 